\title{
Nonlinear Pulse Distortion in Few-Mode Fiber
}

\author{
Naoise Mac Suibhne ${ }^{1, a}$, Regan Watts ${ }^{2}$, Stylianos Sygletos ${ }^{1, b}$, Fatima C Garcia Gunning ${ }^{1, b}$, \\ Lars Grüner-Nielsen ${ }^{3}$, Andrew D. Ellis ${ }^{1, b}$ \\ ${ }^{1}$ Photonic Systems Group, Tyndall National Institute, Lee Maltings, Cork, Ireland \\ naoise.macsuibhne@tyndall.ie \\ also with ${ }^{\mathrm{a} D e p t}$. Electronic Engineering and ${ }^{\mathrm{b}}$ Dept Physics, University College Cork, Ireland \\ ${ }^{2}$ School of Electronic Engineering, Dublin City University, Ireland \\ ${ }^{3}$ OFSDenmark, Priorparken 680, 2605 Brøndby, Denmark
}

\begin{abstract}
Nonlinear pulse propagation in a few mode fiber is experimentally investigated, by measuring temporal and phase responses of the output pulses by use of a frequency discriminator technique, showing that self-phase modulation, dispersion and linear mode-coupling are the dominant effects.
\end{abstract}

\section{Introduction}

Continued growth and demand on optical communication systems has served to ignite an interest in the design and manufacturing of new types of fibers. Recently there has been particular interest shown in the area of few mode fibers $(F M F)^{1}$. These new fibers, along with mode multiplexing, enable the demonstration of higher capacities in optical networks ${ }^{2}$. Multi-mode propagation is not new, with much of the prior work showing that crossmode effects may degrade the signall ${ }^{3,4}$. However, FMF accommodate only 4 to 6 modes, significantly reducing some of the crossmode effects, whilst still providing for higher capacity.

Another advantage of FMF is the larger effective area, enabling higher optical power densities to be launched into the fiber. Given the large walk off between modes of a FMF, it has been assumed that, unlike multi-mode fibers ${ }^{5}$, the nonlinear behavior of each excited mode would be similar to a single mode fiber (SMF) with intra-mode effects prevalent ${ }^{6}$.

In this paper we will show experimental investigations to verify, for the first time to our knowledge, the nonlinear pulse distortions predictions from current standard models that include self-phase modulation (SPM), dispersion and linear mode coupling for each mode. We examined the transmission of short pulses for two modes of a FMF and analyzed the nonlinear contributions. Full field measurements were undertaken to investigate the cause of pulse distortion, with detailed temporal and phase information presented. For a single wavelength signal, we observed that self-phase modulation prevailed by compressing the pulses for both $\mathrm{LP}_{01}$ and $L \mathrm{P}_{02}$, as predicted, while for very high input power levels, pulse splitting occurred and the system became very sensitive to polarization.

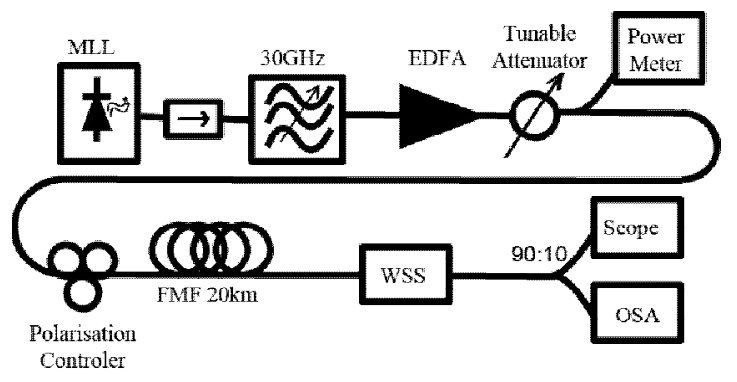

Fig. 1: Experimental Setup

\section{Experiments}

Figure 1 shows the optical setup used to assess the nonlinear pulse propagation in a $20 \mathrm{~km}$ FMF. The $25 \mu \mathrm{m}$-core diameter fiber supported four LP mode groups, with a maximum differential mode delay (DMD) of $2.95 \mathrm{~ns} / \mathrm{km}$, chromatic dispersion between 21.1 and $17.5 \mathrm{ps} / \mathrm{nm} / \mathrm{km}$, and a net loss of less than $10 \mathrm{~dB}$ including launch splice losses. To avoid pulse collisions due to the modal dispersion within the fiber, an actively mode locked laser (MLL) at $1551 \mathrm{~nm}$ was used to launch $\sim 23 p$ s pulses at $10 \mathrm{MHz}$ repetition rate to the FMF, with the aid of a $30 \mathrm{GHz} 3 \mathrm{~dB}$ bandwidth filter.

An EDFA and optical attenuator were used to control the launch power, and the launched pulses were characterized at this point to include any additional distortions from the EDFA. The $\mathrm{LP}_{01}$ and $\mathrm{LP}_{02}$ modes were excited by making a SMF to FMF fusion splice at both ends, which aligned the claddings of both fibers. This launch, close to center, ensured that excitement of $\mathrm{LP}_{11}$ and $\mathrm{LP}_{21}$ was negligible. Though this arrangement was effective for exciting only two modes at the input, it also contributed to the high insertion loss and resulted in an uncertainty over the relative input power ratio between the two modes. It is important to note that the input polarization was adjusted at the highest launch power to give the shortest observable pulse width. 
The output of the FMF was monitored with an oscilloscope via a $50 \mathrm{GHz}$ bandwidth photodetector. In order to recover the phase information, a wavelength selective switch (WSS, see Figure 1) was used as a frequency discriminator. The WSS was programmed to give a linear variation in attenuation as a function of frequency, essentially differentiating the temporal field. Comparing information gathered using the $50 \mathrm{GHz}$ scope with and without the WSS enabled the amplitude and phase information of the pulse to be extracted ${ }^{8}$. Selected measurements were cross-calibrated using a frequency resolved optical gating (FROG) technique and measurements were found to have good agreement with those that were taken using the frequency discriminator. The results were compared to a numerical model which took into account statistic mode coupling between the excited modes.

\section{Results, Analysis and Discussion}
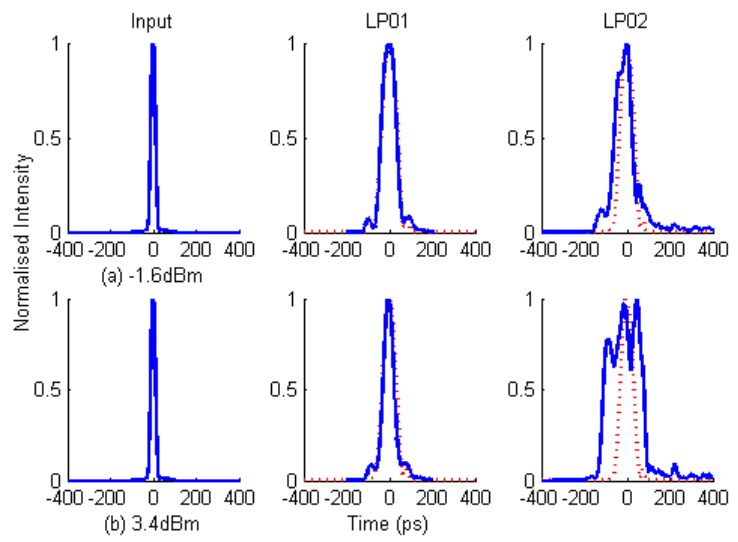

Fig. 2: Input pulse and measured output pulses for $L P_{01}$ and $L P_{02}$ modes for two launched powers.

At low launch powers, two pulses were observed at the fibre output separated by $56.2 \mathrm{~ns}$, in agreement with the calculated DMD taking into account a minimal mode coupling of $\sim 5 \%$. Full field information was recovered for the input signal and for both modes at the output of the $20 \mathrm{~km}$ length of FMF as a function of the launched signal power. Note that no attempt was made to establish the ratio of launched powers between modes. Figure 2 shows examples of pulse temporal profiles for two different powers. The dashed line represents the expected results of the standard FMF numerical model including linear mode coupling and intra-mode nonlinearities, where it was assumed equal launch powers for both modes.

As can be seen from Figure 2, the $\mathrm{LP}_{01}$ closely matches the model, consistent with the validity of the approximations. The numerical model was used to predict the behavior of the $\mathrm{LP}_{02}$ mode and was found to differ considerably from the experiments. This is likely due to an unbalanced ratio of powers between $\mathrm{LP}_{01}$ and $\mathrm{LP}_{02}$.

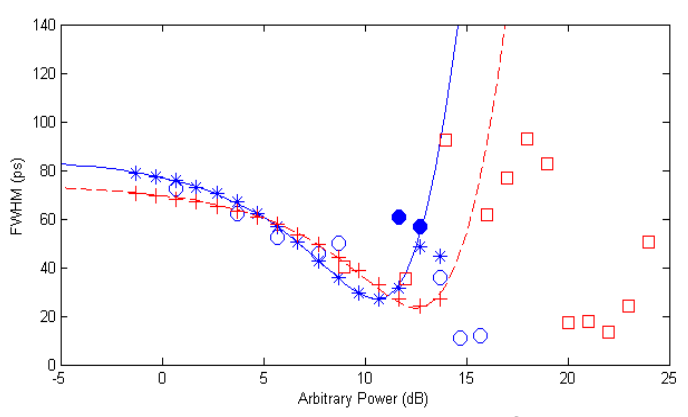

Fig. 3: FWHM width measurements for both $L P_{01}$ (circles) and $\mathrm{LP}_{02}$ (squares)

In order to understand this behavior, the fullwidth at half-maximum (FWHM) of the output pulses were extracted and plotted in Figure 3 against the relative input powers per mode for each of the excited modes. The experimental measurements of $\mathrm{LP}_{01}$ (circles) and $\mathrm{LP}_{02}$ (squares) are compared with FMF model simulations (crosses and asterisks respectively), and also with a one step split-step analysis ${ }^{9}$ (solid and dashed lines respectively). In this case, the FMF is modeled as SPM followed by dispersion, as it would be in an SMF case ${ }^{9,10}$, with the addition of mode coupling, where bandwidth limitations of the experimental components were considered. Here, the mode coupling gives dips in the output pulse width, and the position and amplitude of the dips are power dependent (Figure 3 ). Whether this is a result of nonlinear mode coupling, or of changes in the signal spectrum (wavelength dependent mode coupling), will be the subject of a future investigation.

In Figure 3, for $\mathrm{LP}_{01}$, SPM clearly plays a role by narrowing the pulses as the power increases. As the power is increased further (over $10 \mathrm{~dB}$ ), the nonlinearities induce pulse splitting, and the FWHM measurements begin to increase dramatically. Note that the two solid circle symbols in Figure 3 corresponds to pulse splitting for $\mathrm{LP}_{01}$, and the FWHM shown in these two cases were obtained by measuring the FWHM of the overall pulse by measuring the sum of the two split pulses, considering an even power split. At very high powers, however, the pulse narrowing observed is possibly due to soliton-like effects. Experimental evidence of this is shown in Figure 4, where the evolution of the temporal pulses for $\mathrm{LP}_{01}$ with the increase in the average input power levels is plotted. Clearly pulse splitting, recombining and subsequent narrowing occurs. 


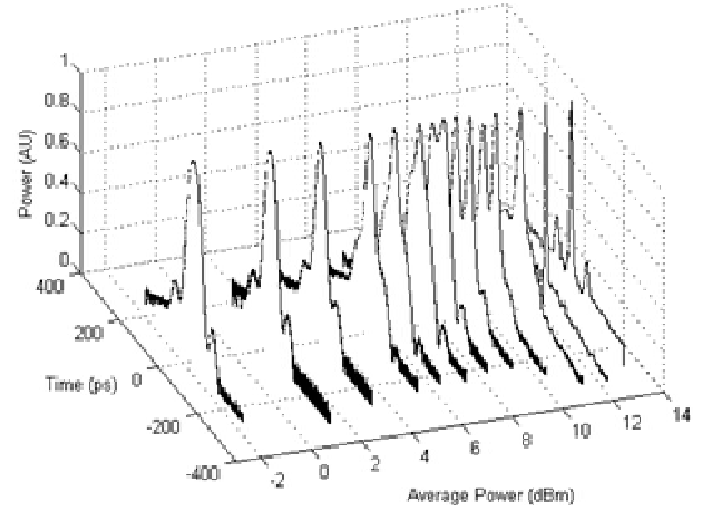

Fig. 4: Evolution of temporal $\mathrm{LP}_{01}$ pulses with average input powers.

The $\mathrm{LP}_{02}$ mode, in turn, also undergoes a similar pulse distortion as $\mathrm{LP}_{01}$, with pulse splitting and broadening being much more pronounced in the experimental results (Figures 2 and 3). This is likely due to a greater power coupling into the $\mathrm{LP}_{02}$ mode, which was estimated to be $\sim 8 \mathrm{~dB}$ higher than the $\mathrm{LP}_{01}$. Taking the mode coupling ratio into account in Figure 3, the numerical predictions closely match the experiments with the pulses strongly affected by SPM. For arbitrary powers above $14 \mathrm{~dB}$, the pulses were highly distorted, generating very scattered results around the predictions. This scattering is likely to be because of additional cross-mode coupling effects. For very high powers, however, where the experimental results are very far from the predictions, we believe that other effects, such as solitons or interference between different degenerate modes, may be playing a role, especially as the measurements were particularly sensitive to polarization.
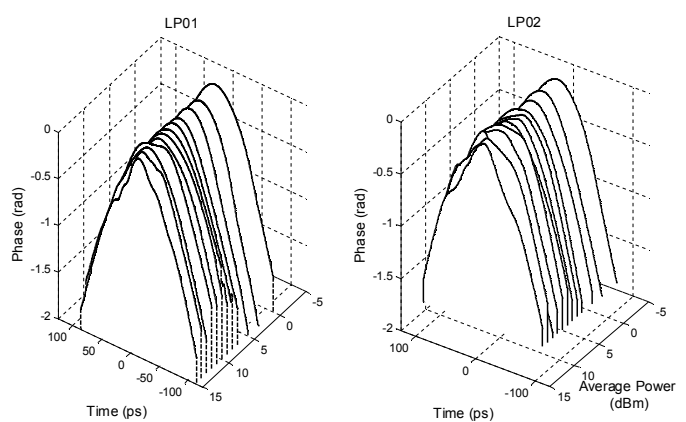

Fig. 5: Phase measured across pulse width for various launch powers. $\mathrm{LP}_{01}$ (left) $\mathrm{LP}_{02}$ (right)

The predicted soliton power for this fiber would be approximately $25 \mathrm{dBm}$ (assuming lossless and 22ps-width pulses) for $\mathrm{LP}_{02}$, increasing to $26 \mathrm{dBm}$ for $\mathrm{LP}_{01}$. Similarly, the soliton period is predicted to be 9.1 and 10.97 $\mathrm{km}$ for $\mathrm{LP}_{01}$ and $\mathrm{LP}_{02}$ modes respectively. Thus, the fiber is approximately two soliton periods long, indicating that soliton propagation may be possible and it will also be the subject of further investigations.

Finally, the evolution of the temporal phase, which was measured experimentally, is plotted for both modes in Figure 5 . For the lowest power pulse, both modes reveal a quadratic phase profile, consistent with propagation being dominated by dispersion. As the temporal pulses split at high powers, the phase begins to depart from the quadratic distribution, due likely to SPM from the individual pulse peaks generated in the pulse splitting.

\section{Conclusions}

The nonlinear propagation of optical pulses is experimentally investigated for the case of a few-mode optical fiber (FMF), in order to demonstrate the validity of current numerical transmission models. A multi-mode propagation model, which included chromatic dispersion, intra-mode nonlinearity and linear cross-mode coupling, was compared with the experimental data, indicating good agreement for both $\mathrm{LP}_{01}$ and $\mathrm{LP}_{02}$ modes. At very high powers, however, the measurements drifted away from the model, and this is likely due to constructive effects from cross-mode coupling and birefringence, or in some cases, the possible presence of soliton effects.

\section{Acknowledgements}

The authors would like to acknowledge Science Foundation Ireland under grant number 06/IN/I969, the Higher Education Authority via the INSPIRE programme, and the EU 7th Framework Programme under grant agreement 228033 (MODE-GAP).

\section{References}

[1] L. Grüner-Nielsen et al., OFC'12 PDP5A.1

[2] S. Randel et al., OFC'12, PDP5C.5

[3] D. Marcuse, "Theory of Dielectric Optical Waveguides", Academic Press, 1974

[4] B. Crosignani et al., Optics Letters, Vol. 6, No.7, Jul 1981

[5] P. L. Baldeck et al., Optics Letters, Vol. 12, No. 8, Aug 1987

[6] F. Ferreira et al., IEEE Photonics Technology Letters, Vol. 24, No. 4, Feb 15, 2012

[7] H. A. Haus, Proceedings of the IEEE, Vol. 81, No. 7, Jul 1993

[8] R. Watts et al., Optics Communications, vol. 285, no. 8, 2012

[9] G. P. Agrawal, "Nonlinear Fiber Optics" $4^{\text {th }}$ Ed., Academic Press, 2007

[10] D. J. Malyon et al., Electronic Letters, Vol. 27, No. 2, January 1991 